\title{
Supply Chain Coordination with Carbon Trading Price and Consumers' Environmental Awareness Dependent Demand
}

\author{
Qinghua Pang (D), Mingzhen Li (D), Tiantian Yang $(\mathbb{D}$, and Yi Shen $(\mathbb{D}$ \\ School of Business Administration, Hohai University, Changzhou 213022, China \\ Correspondence should be addressed to Qinghua Pang; pangqh77@126.com
}

Received 21 July 2017; Revised 13 January 2018; Accepted 24 January 2018; Published 18 March 2018

Academic Editor: Yakov Strelniker

Copyright (c) 2018 Qinghua Pang et al. This is an open access article distributed under the Creative Commons Attribution License, which permits unrestricted use, distribution, and reproduction in any medium, provided the original work is properly cited.

\begin{abstract}
Carbon emissions reduction in supply chain is an effective method to reduce the greenhouse effect. The paper investigates the impacts of carbon trading price and consumers' environmental awareness on carbon emissions in supply chain under the cap-andtrade system. Firstly, it analyzes the centralized decision structure and obtains the requirements to coordinate carbon emissions reduction and order quantity in supply chain. Secondly, it proposes the supply chain coordination mechanism with revenue-sharing contract based on quantity discount policy, and the requirements that the contract parameters need to satisfy are also given. Thirdly, assuming the market demand is affected by consumer's environmental awareness in addition form, the paper proposes the methods to determine the optimal order quantity and the optimal level of carbon emissions through model optimization. Finally, it investigates the impacts of carbon trading price on carbon emissions in supply chain. The results show that clean manufacturer's optimal per-unit carbon emissions increase as the carbon trading price increases, while nongreen manufacturer's optimal per-unit carbon emissions decrease as the carbon trading price increases. For the middle emissions manufacturer, the optimal per-unit carbon emissions depend on the relationship between the carbon trading price and the carbon reduction coefficient.
\end{abstract}

\section{Introduction}

To control and reduce carbon emissions is a global problem. With "United Nations Framework Convention on Climate Change" and "Kyoto Protocol" signed and coming into force, carbon emissions reduction and the cap-and-trade system have become the consensus and inevitable trend. Enterprises are the main bodies of carbon emissions reduction; however the development of modern market economy makes any enterprise be placed in some supply chain systems. Then if we control carbon emissions from the perspective of single enterprise, the spillover effects generated by other enterprises' carbon emissions control in the supply chain will be ignored. Only when we solve the problem of carbon emissions reduction cooperation and coordination among supply chain enterprises effectively can we fundamentally promote carbon emissions reduction work. Otherwise it will be difficult for us to achieve the goal of carbon emissions reduction.

With the improvement of cap-and-trade system, the carbon trading price, as the most direct market signal, will regulate the manufacturer's carbon emissions in his production process, thereby affecting the manufacturer's perunit carbon emissions. For example, Baosteel Group, one of the largest steel enterprises in China, has launched two carbon trading projects from 2007 to 2012, aiming to reduce per-unit carbon emissions through energy saving and emissions reduction technologies [1]. Then Baosteel Group sold excess carbon credits at the appropriate price in the carbon trading market and profited more than 150 million RMB [2]. In addition, with the development of social civilization and low-carbon education, there are more environmentally conscious consumers who prefer low-carbon products. How to meet consumers' low-carbon preference has become a key guarantee to win over in the fierce market competition [3]. Therefore, it is of great value and practical significance to study the carbon emissions reduction in the supply chain with considering carbon trading price and consumers' environmental awareness (CEA).

The investigated supply chain in this paper consists of one manufacturer and one retailer. At the beginning of production, the manufacturer obtains an initial carbon emissions 
limit from the government that has established a carbon emissions center. The manufacturer can buy carbon emissions rights from the carbon emissions center if he exceeds his initial carbon emissions limit at the end of production cycle; otherwise, he can sell his unused carbon emissions rights to the carbon emissions center. The manufacturer also can upgrade his production technology to reduce carbon emissions; then he needs to bear the cost of carbon emissions reduction. The consumers have environmental awareness, which means the products' carbon emissions will influence the market demand. Other assumptions are similar to the newsvendor model. Our research question is that how the carbon trading price and CEA affect carbon emissions reduction in supply chain under cap-and-trade system. We also discuss whether the low-carbon supply chain can be coordinated by the revenue-sharing contract or not; if not, then how to improve the revenue-sharing contract to coordinate the supply chain? Our contributions with respect to the previous literature in this field are that we take both the carbon trading price and CEA into account and also propose the coordination mechanism in supply chain carbon emissions reduction under cap-and-trade system.

The rest of the paper is organized as follows: Section 2 includes literature review. Section 3 introduces model assumptions and notations. Section 4 studies carbon emissions reduction coordination based on revenue-sharing contract. Section 5 optimizes carbon emissions reduction coordination model. Section 6 analyzes impacts of carbon trading price on carbon emissions and Section 7 provides a light numerical experiment. Section 8 summarizes this paper.

\section{Literature Review}

This paper relates to three major research areas, which are supply chain carbon footprint evaluation and management, decision problem of carbon emissions reduction in supply chain, impacts of CEA on carbon emissions reduction, and supply chain coordination. We review some recent representative works in the literature as follows.

It is a basic work to evaluate and manage supply chain's carbon footprint to control carbon emissions in supply chain. Braithwaite and Knivett [4] proposed a methodology to evaluate supply chain's carbon footprint by introducing the "carbon-to-serve" concept. Cholette and Venkat [5] found that different supply chain configurations can result in vastly different energy and emissions' profiles by using a web-based tool to calculate carbon emissions. Sundarakani et al. [6] examine the carbon footprint across supply chains and use transport methods to construct the analytical model. Lam et al. [7] presented a new method for supply chain synthesis and used a demand-driven approach to assess the feasible ways for transferring energy from renewable sources to customers in a given region. $\mathrm{Xu}$ and Fan [8] established the carbon footprint calculation models of fixed emissions sources and dynamic emissions sources in the supply chain. Compared with these surveyed papers, our work is based on these studies and focuses on the impacts of carbon trading price and CEA on carbon emissions reduction.
In the carbon emissions reduction literatures, the decision problem of carbon emissions reduction in supply chain is a hot topic. Benjaafar et al. [9] illustrated how carbon emission concerns could be integrated into operational decisionmaking and analyzed the impact of operational decisions on carbon emissions. Rosič and Jammernegg [10] extended the dual sourcing model based on the newsvendor framework by considering the environmental impact of transport with emission taxes. Song and Leng [11] investigated the classical single-period problem under different carbon emissions policies. Du et al. [12] focused on the impact of "cap-and-trade" mechanism, proposed a game-theoretical analytical model, and derived a unique Nash equilibrium. Jaber et al. [13] presented a supply chain coordination model while accounting for greenhouse gas emissions from manufacturing processes. $\mathrm{Xu}$ et al. [14] proposed three differential game models with the emission reduction of the product and the retailer's promotion dependent demand. $\mathrm{Li}$ et al. [15] proposed a carbon trading model discussed between enterprises under strict carbon cap. Zhao et al. [16] developed a retailer-driven revenue-sharing contract to coordinate the supply chain with the constraint of product carbon emissions. Lu and Chen [17] studied the supply chain coordination with buyback contract under different carbon emissions policies and found buyback contract can coordinate supply chain. Compared with the surveyed papers in this scope, our work focuses on the coordination mechanism about carbon emissions reduction and order quantity in the supply chain.

CEA affects market demand, which has an impact on the enterprise's carbon emissions. Liu et al. [18] found that as CEA increases, retailers and manufacturers with superior ecofriendly operations will benefit. Zhang et al. [19] analyzed the impact of CEA on order quantities and channel coordination, in which the manufacturer produces the environmental products and the traditional products. Wang and Zhao [20] studied how to determine the optimum order quantity and which situation the supplier should choose to reduce carbon emissions. Xie et al. [21] studied carbon emission reduction and sharing decision-making of supply chain system with the carbon emissions level dependent demand. Chen [22] proposed several important topics for future research based on a brief overview of the current research in the field of low-carbon supply chain management, including the impacts of CEA on carbon emissions reduction. Compared with the literatures in this scope, our work not only considers the CEA, but also considers the carbon trading price.

Through coordination, supply chain can fully exploit its potential and improve its performance [23]. There are many works in the field of supply chain coordination. Heydari and Norouzinasab [24] proposed a coordination mechanism to coordinate both pricing and ordering decisions simultaneously based on quantity discount. Chakraborty and Chatterjee [25] developed the economics of surcharge pricing as a supply chain coordinating mechanism under JIT environment. Heydari [26] proposed a model to coordinate replenishment decisions in a two-stage supply chain by considering truckload limitation based on delay in payments. Feng et al. [27] proposed a revenue-sharing-and-buy-back contract to coordinate two-stage supply chain in which 


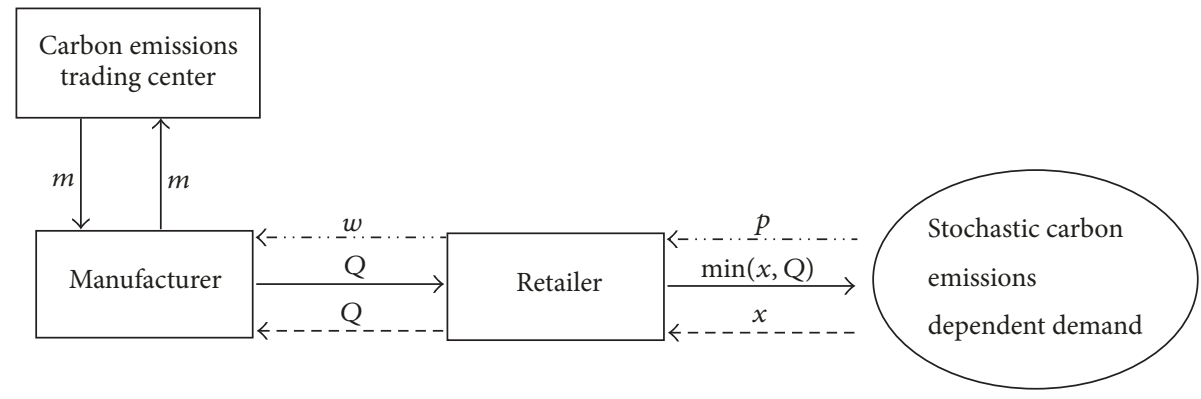

FIGURE 1: Investigated low-carbon supply chain.

members experience budget constraints. Heydari et al. [28] proposed a two-level delay-in-payments contract to coordinate both ordering and marketing decisions with stochastic credit-dependent demand. Ebrahimi et al. [29] proposed a delay in payment contract to coordinate a periodic review supply chain with stochastic promotional effort dependent demand. Compared with the surveyed papers in this scope, our work focuses on the coordination mechanism of lowcarbon supply chain, including carbon emissions reduction coordination and commercial coordination.

The above-mentioned literatures all carry out in-depth studies on the supply chain carbon emissions reduction. They put forward many carbon emissions control methods and models, which are a great contribution to the field of carbon emissions reduction. Meanwhile it is also needed to be pointed out that there are three issues that should be explored in depth: (1) There are few literatures concerning the impacts of carbon trading price on the supply chain carbon emissions reduction. In fact, carbon trading price has important impact on the supply chain carbon emissions reduction and the carbon emissions allocation. (2) There are also few literatures that take both the carbon trading price and CEA into account. It has become an important problem to study the impacts of carbon trading price on supply chain carbon emissions reduction while taking CEA into account. (3) It is necessary to further study the coordination mechanism in supply chain carbon emissions reduction. Under the constraint of carbon emissions, if the supply chain members try to optimize their own profits to reduce their carbon emissions, carbon emissions reduction of the whole supply chain will often not be the optimal; that is, there is a "marginal double" effect. So it is also needed to explore how to achieve the supply chain carbon emissions reduction coordination.

In the paper, we discuss the impacts of carbon trading price and CEA on the supply chain carbon emissions reduction with stochastic demand, and revenue-sharing contract is used to coordinate the supply chain carbon emissions reduction, which will provide some help and advice for supply chain carbon emissions reduction in real world.

\section{Model Description and Notations}

The supply chain studied in this paper includes one manufacturer and one retailer. The manufacturer processes the raw material into products and sells them to the retailer at wholesale price $w$, and the retailer sells the products to the consumers who have environmental awareness at the perunit sale price $p$. Before the sale season, the retailer has only one chance to buy products from the manufacturer and the manufacturer has the ability to produce the products that the retailer orders. The retailer faces a stochastic CEA dependent demand. All members are risk neutral, and information is symmetric among them. The investigated low-carbon supply chain in this paper is shown in Figure 1.

Assume that the government has established a carbon emissions trading center and the enterprises buy or sell carbon emissions rights just for normal production; namely, there is no speculation. At the beginning of production cycle, the manufacturer obtains an initial carbon emissions limit from the government. At the end of production cycle, if the manufacturer's total carbon emissions exceed the initial carbon emissions limit, he needs to buy carbon emissions rights from the carbon emissions trading center at the perunit carbon trading price; if the manufacturer's total carbon emissions do not exceed the initial carbon emissions limit, he can sell the carbon emissions rights to the carbon emissions trading center at the per-unit carbon trading price.

As the consumers pay more attention to the environmental change, low-carbon products are more and more welcomed by the market. The products' carbon emissions affect the market demand, which also affect the supply chain members' market competitiveness. So it is necessary for supply chain members to upgrade their production technology to reduce their carbon emissions. Assume the manufacturer has the ability to reduce his products' carbon emissions. Before the manufacturer decides to upgrade his production technology, the initial carbon emissions per-unit product is $e_{o}$. When the manufacturer decides to carry out technological upgrading for carbon emissions reduction, the carbon emissions per-unit product is $e\left(0<e \leq e_{o}\right)$. The manufacturer needs to bear the cost of carbon emissions reduction when he wants to upgrade his production technology. According to the literatures $[1,3,14,20]$, we can use $\alpha\left(e_{o}-e\right)^{2} / 2$ as the cost of carbon emissions reduction; here $\alpha(\alpha>0)$ is the cost coefficient of the manufacturer's carbon emissions reduction.

The following notations are applied in the mathematical models. 
$M$ : the initial carbon emissions limit of the manufacturer

$m$ : the per-unit carbon trading price

$e_{o}$ : the initial carbon emissions per-unit product

$e$ : the carbon emissions per-unit product after technological upgrading $\left(0<e \leq e_{o}\right)$ (decision variable)

$x$ : stochastic market demand when the carbon emissions per-unit product is $e$

$\mu$ : mean value of $x$

$f(x \mid e)$ : probability density function of $x$

$F(x \mid e)$ : differentiable cumulated distribution function of $x$, which is continuously differentiable

$p$ : the retail price

$w$ : the wholesale price

Q: order quantity (decision variable)

$c_{i}$ : supply chain members' marginal unit production cost $(i=r, m)$ and $c=c_{r}+c_{m}$

$g_{i}$ : supply chain members' marginal unit goodwill penalty cost $(i=r, m)$ and $g=g_{r}+g_{m}$

$v$ : salvage value for unsold unit product $(v<c)$.

Then the expectation sale quantity is $S(Q)=$ $E \min (Q, x)=Q-\int_{0}^{Q} F(x \mid e) d x$; the expected unsold quantity is $I(Q)$ and $I(Q)=Q-S(Q)$; the expected out of stock is $L(Q)$ and $L(Q)=\mu-S(Q)$. In particular, it is reasonable that $p>c>v, p>c+m e, c_{i}>0, g_{i}>0(i=r, m)$. In addition, we also assume that $\alpha f(x \mid e)(p-v+g)>m^{2}$.

\section{Coordination Model of Carbon Emissions Reduction}

To design supply chain coordination mechanism, we study the centralized structure firstly. In this condition, all supply chain members are managed by one central manager; then we can get the maximum profit of centralized supply chain as the goal to coordinate supply chain. This profit is defined as sum of profits of the manufacturer and retailer, which is be described as

$$
\begin{aligned}
& \prod_{t}(Q, e)=\prod_{m}(Q, e)+\prod_{r}(Q)=\left[w Q-c_{m} Q\right. \\
& \left.\quad-g_{m} L(Q)-m(e Q-M)-\frac{1}{2} \alpha\left(e_{o}-e\right)^{2}\right] \\
& +\left[p S(Q)+v I(Q)-w Q-c_{r} Q-g_{r} L(Q)\right] \\
& =p S(Q)+v I(Q)-g L(Q)-c Q-m(e Q-M) \\
& -\frac{1}{2} \alpha\left(e_{o}-e\right)^{2}=(p-v+g) S(Q)-(c-v+m e) Q \\
& +m M-g \mu-\frac{1}{2} \alpha\left(e_{o}-e\right)^{2} .
\end{aligned}
$$

From (1), we can obtain the Hessian matrix of $\prod_{t}(Q, e)$, which can be written as $A$.

$$
\begin{aligned}
A & =\left[\begin{array}{cc}
\frac{\partial^{2} \prod_{t}(Q, e)}{\partial Q^{2}} & \frac{\partial^{2} \prod_{t}(Q, e)}{\partial Q \partial e} \\
\frac{\partial^{2} \prod_{t}(Q, e)}{\partial e \partial Q} & \frac{\partial^{2} \prod_{t}(Q, e)}{\partial e^{2}}
\end{array}\right]=(p-v+g) \\
& \cdot\left[\begin{array}{cc}
-f(Q \mid e) & R \\
R & -f(Q \mid e)\left(\frac{\partial Q}{\partial e}\right)^{2}-\frac{2 m(\partial Q / \partial e)+\alpha}{p-v+g}
\end{array}\right] .
\end{aligned}
$$

Here $R=-f(Q \mid e)(\partial Q / \partial e)-m /(p-v+g)$. From the matrix $A$, we can see that $\partial^{2} \prod_{t}(Q, e) / \partial Q^{2}=-f(Q \mid e)(p-$ $v+g)<0 . \partial^{2} \prod_{t}(Q, e) / \partial e^{2}=-f(Q \mid e)(\partial Q / \partial e)^{2}(p-v+g)-$ $2 m(\partial Q / \partial e)-\alpha=-(1 / \alpha)\left[\alpha f(Q \mid e)(p-v+g)(\partial Q / \partial e)^{2}+\right.$ $\left.2 m \alpha(\partial Q / \partial e)-\alpha^{2}\right]<-(1 / \alpha)\left[m^{2}(\partial Q / \partial e)^{2}+2 m \alpha(\partial Q / \partial e)+\right.$ $\left.\alpha^{2}\right]=-(1 / \alpha)(m(\partial Q / \partial e)+\alpha)^{2}<0$ due to $\alpha f(x \mid e)(p-v+$ g) $>m^{2}$; namely, $\partial^{2} \prod_{t}(Q, e) / \partial e^{2}<0$. We also can get that $|A|=(p-v+g)^{2}\left[f(Q \mid e)\left(f(Q \mid e)(\partial Q / \partial e)^{2}-(2 m(\partial Q / \partial e)+\right.\right.$ $\left.\alpha) /(p-v+g))-R^{2}\right]=\alpha f(Q \mid e)(p-v+g)-m^{2}>0$ due to $\alpha f(x \mid e)(p-v+g)>m^{2}$. In a word, we can get that $\partial^{2} \prod_{t}(Q, e) / \partial Q^{2}<0, \partial^{2} \prod_{t}(Q, e) / \partial e^{2}<0$ and $|A|>0$, which means $\prod_{t}(Q, e)$ is the concave function of both $Q$ and $e$.

For the supply chain system, we should determine the optimal order quantity $Q^{*}$ and the optimal carbon emissions per-unit product $e^{*}$ to maximize $\prod_{t}(Q, e)$. Then $Q^{*}$ and $e^{*}$ should satisfy $\partial \prod_{t}\left(Q^{*}, e\right) / \partial Q=0$ and $\partial \prod_{t}\left(Q, e^{*}\right) / \partial e=0$, respectively; then we get (3). Obviously, (3) is the benchmark for other conditions to coordinate the supply chain.

$$
\begin{array}{r}
F\left(Q^{*} \mid e\right)-\frac{p-c+g-m e}{p-v+g}=0, \\
(p-v+g) \frac{\partial S(Q)}{\partial e}-m Q+\alpha\left(e_{o}-e^{*}\right)=0 .
\end{array}
$$

In reality, the retailer or the manufacturer makes decisions from the view of optimizing his own profit, which is the most common condition called decentralized structure. In decentralized structure, the profit function of the retailer and the manufacturer can be written respectively as

$$
\begin{aligned}
\prod_{r}^{d}(Q)= & p S(Q)+v I(Q)-c_{r} Q-w Q-g_{r} L(Q) \\
= & \left(p-v+g_{r}\right) S(Q)-\left(c_{r}+w-v\right) Q \\
& -g_{r} \mu \\
\prod_{m}^{d}(Q, e)= & w Q-c_{m} Q-g_{m} L(Q)-m(e Q-M) \\
& -\frac{1}{2} \alpha\left(e_{o}-e\right)^{2} \\
= & g_{m} S(Q)-\left(c_{m}-w+m e\right) Q+m M \\
& -g_{m} \mu-\frac{1}{2} \alpha\left(e_{o}-e\right)^{2} .
\end{aligned}
$$

In (4), $p S(Q), v I(Q), c_{r} Q, w Q$, and $g_{r} L(Q)$, respectively, are the retailer's sales income, income of unsold unit product, 
cost of marketing, goodwill penalty, and transfer payment. In (5), $w Q, c_{m} Q, g_{m} L(Q), m(e Q-M)$, and $-1 / 2 \alpha\left(e_{o}-e\right)^{2}$, respectively, are the manufacturer's sales income, cost of production, cost (if $e Q>M$ ) of buying the carbon emissions rights or income (if $e Q<M$ ) of selling the carbon emissions rights, and cost of carbon emissions reduction.

From (4), we can get $\partial^{2} \prod_{r}^{d}(Q) / \partial Q^{2}=-f(Q \mid e)(p-v+$ $\left.g_{r}\right)<0$, which means that $\prod_{r}^{d}(Q)$ is the concave function of $Q$. So if the retailer wants to maximize his profit, his optimal order quantity $Q_{r}^{*}$ should satisfy $\partial \prod_{r}^{d}\left(Q_{r}^{*}, e\right) / \partial Q=0$; similarly, $\partial^{2} \prod_{m}^{d}(Q, e) / \partial e^{2}=-[1-F(Q \mid e)](\partial Q / \partial e)^{2}-$ $\alpha<0$, which means that $\prod_{m}^{d}(Q, e)$ is the concave function of $e$. If the manufacturer wants to maximize his profit, his optimal carbon emissions per-unit product should satisfy $\partial \prod_{m}^{d}\left(Q, e_{m}^{*}\right) / \partial e=0$. Then we can get

$$
\begin{aligned}
F\left(Q_{r}^{*} \mid e\right)-\frac{p-c_{r}+g_{r}-w}{p-v+g_{r}} & =0, \\
g_{m} \frac{\partial S(Q)}{\partial e}-m Q+\alpha\left(e_{o}-e_{m}^{*}\right) & =0 .
\end{aligned}
$$

In decentralized structure, if the supply chain is also in the optimal condition, we can get $F\left(Q^{*} \mid e\right)=F\left(Q_{r}^{*} \mid e\right)$ based on (3) and (6). From $F\left(Q^{*} \mid e\right)=F\left(Q_{r}^{*} \mid e\right)$, we can get $w=\left(\left(p-v+g_{r}\right) /(p-v+g)\right)(c-v+m e)-c_{r}+v \leq c-v+m e-c_{r}+v$; that is, $w \leq c_{m}+m e$. Meanwhile, for the rational manufacturer, there should be $w>c_{m}$ before technological upgrading and $w>c_{m}+m e$ (i.e., the wholesale price should be greater than the sum of the production cost and the cost of carbon emissions rights purchased) after technological upgrading in order to guarantee his own profit. So the supply chain is not the optimal in decentralized structure; that is, $F\left(Q^{*} \mid e\right) \neq$ $F\left(Q_{r}^{*} \mid e\right)$, which means that the optimal order quantity $Q_{r}^{*}$ of the retailer in decentralized structure is not equal to the optimal order quantity $Q^{*}$ of the supply chain in centralized structure (in fact, $Q_{r}^{*}<Q^{*}$ ). Similarly, we also can conclude that the optimal carbon emissions per-unit product $e_{m}^{*}$ of the manufacturer in decentralized structure is not equal to the optimal carbon emissions per-unit product $e^{*}$ of the supply chain in centralized structure (in fact, $e_{m}^{*}>e^{*}$ ).

This indicates that the decision-making of the supply chain members in decentralized structure is not optimal. There are two "marginal double" problems: one is the order quantity $\left(Q_{r}^{*}<Q^{*}\right)$; the other is the carbon emissions perunit product $\left(e_{m}^{*}>e^{*}\right)$. So some measures need to be taken, such as Pareto improving contract [30], to coordinate the low-carbon supply chain in both order quantity and carbon emissions reduction to achieve Pareto improvement.

Revenue-sharing contract is a kind of contract to coordinate supply chain. The contract can be described by two parameters $(w, \phi)$ : the manufacturer charges the retailer a unit wholesale price $w$, lower than the unit marginal cost $c_{m}$, in exchange for a percentage $(1-\phi)$ of the retailer's revenue. The position $w<c_{m}$ guarantees channel coordination whereas $\phi$ determines the distribution of total profits between the manufacturer and the retailer. Particularly, $\phi$ is the supply chain profit share gained by the retailer. With revenuesharing contract, the profit function of the retailer can be rewritten as

$$
\begin{aligned}
\prod_{r}^{c}(Q)= & \phi[p S(Q)+v I(Q)]-c_{r} Q-w Q-g_{r} L(Q) \\
= & {\left[\phi(p-v)+g_{r}\right] S(Q)-\left(c_{r}+w-\phi v\right) Q } \\
& -g_{r} \mu .
\end{aligned}
$$

In (7), $\phi[p S(Q)+v I(Q)]$ is the percentage of income that the retailer keeps; the rest is the same as (4). Then the profit of the manufacturer is equal to the sum of (5) and $(1-\phi)[p S(Q)+v I(Q)]$. Assume $Q_{r}^{c *}$ is the optimal order quantity of the retailer in revenue-sharing contract and $e_{m}^{c *}$ is the optimal carbon emissions per-unit product. From (7), we can get $F\left(Q_{r}^{c *} \mid e\right)=\left(\phi p+g_{r}-c_{r}-w\right) /\left(\phi(p-v)+g_{r}\right)$. Due to $w>c_{m}+m e,(1-\phi)(p-v)(c+m e)+g_{m}(c+m e)+v\left(\phi g-g_{r}\right)>0$, so $\left(\phi p+g_{r}-c_{r}-w\right) /\left(\phi(p-v)+g_{r}\right)<(p-c+g-$ $m e) /(p-v+g)$, which means $F\left(Q_{r}^{c *} \mid e\right)<F\left(Q^{*} \mid e\right)$. That is to say, the optimal order quantity of the retailer with revenue-sharing contract is still less than the optimal order quantity of the supply chain in centralized structure $\left(Q_{r}^{c *}<\right.$ $\left.Q^{*}\right)$. Similarly, we can get that the optimal carbon emissions per-unit product of the manufacturer with revenue-sharing contract is still higher than the optimal carbon emissions per-unit product of the supply chain in centralized structure $\left(e_{m}^{c *}>e^{*}\right)$. This means that the classical revenue-sharing contract cannot coordinate the supply chain.

There are some reasons to explain this phenomenon: When the manufacturer offers the revenue-sharing contract $(w, \phi)$ and decides his optimal carbon emissions level, the retailer's order quantity will be less than the optimal order quantity of the supply chain because the demand is influenced by the products' carbon emissions and $w$ is a set wholesale price. Because order quantity of retailer is less, the manufacturer will choose the carbon emissions per-unit product that can maximize his own profit, rather than the optimal carbon emissions per-unit product of the supply chain. Then the classical revenue-sharing contract cannot coordinate the lowcarbon supply chain. So it needs to improve classical revenuesharing contract to achieve Pareto improvement.

Maybe it is a way that the retailer shares the manufacturer's carbon emissions reduction cost to coordinate supply chain. But, in many cases, it is more feasible that only one supply chain member bears the carbon emissions reduction cost. Therefore, in this paper, we also assume the manufacturer bears the carbon emissions reduction cost alone. Here, we introduce the quantity discount policy into revenuesharing contract. That is, the wholesale price that the supplier provides to the manufacturer varies with the manufacturer's order quantity, and the revenue-sharing contract parameters are $(w(Q), \phi)$. Then, in this condition, the profit function of the retailer and the manufacturer can be rewritten as (8)-(9), respectively.

$$
\begin{aligned}
\prod_{r}^{c}(Q)= & \phi[p S(Q)+v I(Q)]-c_{r} Q-w(Q) Q \\
& -g_{r} L(Q) \\
= & {\left[\phi(p-v)+g_{r}\right] S(Q) } \\
& -\left(c_{r}+w(Q)-\phi v\right) Q-g_{r} \mu,
\end{aligned}
$$




$$
\begin{aligned}
\prod_{m}^{c}(Q, e)= & (1-\phi)[p S(Q)+v I(Q)]-c_{m} Q \\
& +w(Q) Q-g_{m} L(Q)-m(e Q-M) \\
& -\frac{1}{2} \alpha\left(e_{o}-e\right)^{2} \\
= & {\left[(1-\phi)(p-v)+g_{m}\right] S(Q) } \\
& -\left(c_{m}-w(Q)-(1-\phi) v+m e\right) Q \\
& -g_{m} \mu-\frac{1}{2} \alpha\left(e_{o}-e\right)^{2}+m M .
\end{aligned}
$$

In $(9),(1-\phi)[p S(Q)+v I(Q)]$ is the percentage of income that the retailer transfers to the manufacturer; the rest is the same as (5). If the improved revenue-sharing contract can coordinate the supply chain, the retailer's order quantity and the manufacturer's carbon emissions per-unit product should equal the optimal order quantity and the carbon emissions per-unit product of the supply chain, respectively; that is, they should satisfy $\partial \prod_{r}^{c}\left(Q^{*}\right) / \partial Q=\partial \prod_{t}\left(Q^{*}, e\right) / \partial Q$ and $\partial \prod_{m}^{c}(Q, e) / \partial e=\partial \prod_{t}(Q, e) / \partial e$. From (3) and (8)-(9), we can get

$$
\begin{aligned}
\frac{c_{r}+w(Q)-\phi v}{\phi(p-v)+g_{r}} & =\frac{c-v+m e}{p-v+g}, \\
\frac{m Q-\alpha\left(e_{o}-e_{m}\right)-Q \partial w / \partial e}{(1-\phi)(p-v)+g_{m}} & =\frac{m Q-\alpha\left(e_{o}-e_{m}\right)}{p-v+g} .
\end{aligned}
$$

From (10), we can get

$$
\begin{aligned}
w(Q)= & \frac{\phi(p-v)+g_{r}}{p-v+g}(c-v+m e)-c_{r}+\phi v \\
& +\frac{\phi(p-v)+g_{m}}{p-v+g} \frac{(1 / 2) \alpha\left(e_{o}-e_{m}\right)^{2}-m M}{Q}
\end{aligned}
$$

Substituting (11) into (8)-(9), then (12) can be obtained.

$$
\begin{aligned}
\prod_{r}^{c}(Q)= & \frac{\phi(p-v)+g_{r}}{p-v+g}\left(\prod_{t}(Q, e)+g \mu\right)-g_{r} \mu \\
\prod_{m}^{c}(Q, e)= & \frac{(1-\phi)(p-v)+g_{m}}{p-v+g}\left(\prod_{t}(Q, e)+g \mu\right) \\
& -g_{m} \mu .
\end{aligned}
$$

From (12), it can be seen that the profit functions of the supply chain members are all dependent functions of the whole supply chain's profit function, which means the retailer's order quantity and the manufacturer's carbon emissions per-unit product equal the optimal order quantity and the carbon emissions per-unit product of the supply chain. So, in this condition, the improved revenue-sharing contract can coordinate the supply chain, including order quantity and carbon emissions, which achieves the dual goal of economic development and carbon emissions reduction. It also should be pointed out that if the supply chain members are willing to use the improved revenue-sharing contract to coordinate supply chain in reality, their profits should not be less than the profit in decentralized supply chain. Therefore we should consider the participation constraint in supply chain practice. Namely, for the retailer and the manufacturer, their profit should, respectively, satisfy $\prod_{r}^{c}(Q) \geq \prod_{r}^{d}(Q)$ and $\prod_{m}^{c}(Q, e) \geq \prod_{m}^{d}(Q, e)$. Here, the superscript $c$ and $d$, respectively, mean the improved revenue-sharing contract structure and decentralized structure.

\section{Model Optimization}

From the discussion mentioned above, we can come to a conclusion that the traditional revenue-sharing contract cannot coordinate low-carbon supply chain, while the improved revenue-sharing contract based on quantity discount policy can coordinate low-carbon supply chain. In this section, we propose the methods to determine the optimal order quantity and the optimal level of carbon emissions on the basis of the hypothesis that the demand and the consumers' low-carbon preference satisfy addition form.

Because the demand is stochastic and dependent on the products' carbon emissions, suppose the market demand $X(e, \xi)$ is the function of random factor $\xi$ and per-unit product's carbon emissions $e$, and $\xi$ is independent of $e$; $f(\xi)$ and $F(\xi)$ are the probability density function and the differentiable cumulated distribution function of $\xi$, respectively. Usually we can use two forms to describe how the carbon emissions affect the demand: addition form and multiplication form. In this paper, suppose $X(e, \xi)=y(e)+\xi$. Because the influence of products' carbon emissions on market demand is diminishing marginal utility, we can suppose $y(e)$ is the monotone increasing and concave function of the per-unit product's carbon emissions $e$; namely, $y^{\prime}(e)<0$, $y^{\prime \prime}(e) \geq 0$.

When the market demand satisfies $X(e, \xi)=y(e)+\xi$, we can get

$$
\begin{aligned}
S(Q) & =Q-\int_{0}^{Q} F(x \mid e) d x \\
& =Q-\int_{y(e)}^{Q} F(x-y(e)) d x \\
& =Q-\int_{0}^{Q-y(e)} F(t) d t .
\end{aligned}
$$

Substituting (13) into (1), then (1) can be rewritten as

$$
\begin{aligned}
\prod_{t}(Q, e)= & p S(Q)+v I(Q)-g L(Q)-c Q \\
& -m(e Q-M)-\frac{1}{2} \alpha\left(e_{o}-e\right)^{2} \\
= & (p-c+g-m e) Q \\
& -(p-v+g) \int_{0}^{Q-y(e)} F(t) d t+m M \\
& -g \mu-\frac{1}{2} \alpha\left(e_{o}-e\right)^{2} .
\end{aligned}
$$


As we have proved that $\prod_{t}(Q, e)$ is the concave function of both $Q$ and $e$ in Section 4, if the carbon emissions perunit product $e$ is given, the optimal order quantity $Q^{*}$ should satisfy

$$
\begin{aligned}
\frac{\partial \prod_{t}\left(Q^{*}, e\right)}{\partial Q}= & (p-c+g-m e) \\
& -(p-v+g) F\left(Q^{*}-y(e)\right)=0 .
\end{aligned}
$$

From (15), we can get

$$
Q^{*}=Q^{*}(e)=y(e)+F^{-1}\left(\frac{p-c+g-m e}{p-v+g}\right) \text {. }
$$

Substituting (16) into (13), then (14) can be rewritten as

$$
\begin{aligned}
\prod_{t}\left(Q^{*}, e\right)= & \omega Q^{*}-(p-v+g) \int_{0}^{Q^{*}-y(e)} F(t) d t \\
& +m M-g \mu-\frac{1}{2} \alpha\left(e_{o}-e\right)^{2} \\
= & \omega\left[y(e)+F^{-1}\left(\frac{\omega}{p-v+g}\right)\right] \\
& -(p-v+g) \int_{0}^{F^{-1}(\varpi /(p-v+g))} F(t) d t \\
& +m M-g \mu-\frac{1}{2} \alpha\left(e_{o}-e\right)^{2} .
\end{aligned}
$$

In (17), $\omega=p-c+g-m e$. Then the optimal per-unit product's carbon emissions $e^{*}$ should satisfy $\partial \prod_{t}\left(Q^{*}, e^{*}\right) / \partial e=0$; that is, $e^{*}$ should satisfy

$$
\begin{aligned}
& m\left[y\left(e^{*}\right)+F^{-1}\left(\frac{p-c+g-m e^{*}}{p-v+g}\right)\right] \\
& \quad=\left(p-c+g-m e^{*}\right) y^{\prime}\left(e^{*}\right)+\alpha\left(e_{o}-e^{*}\right) .
\end{aligned}
$$

According to (16) and (18), we can determine the optimal order quantity and the optimal per-unit product's carbon emissions. However, both (16) and (18) are relatively complex and we cannot intuitively observe the impacts of carbon trading price on carbon emissions, so the relevant functions need further refining. Suppose $y(e)=\beta-\gamma e$, here $\beta$ is the basic market demand, $\gamma(\gamma>0)$ is the sensitive coefficient of the market demand to the per-unit product's carbon emissions, and $\beta>\gamma e_{o}$. $\xi$ satisfies uniform distribution in $[A, B]$, and $A>0, B>0$. So the probability density function and the differentiable cumulated distribution function of $\xi$ are $f(\xi)=1 /(B-A)$ and $F(\xi)=(\xi-A) /(B-A)$, the mean value is $\mu=(A+B) / 2$, and the variance is $\sigma^{2}=(B-A)^{2} / 12$, $F^{-1}(\xi)=A+\xi(B-A)$. The optimal order quantity and the optimal per-unit product's carbon emissions of the supply chain can be rewritten as (19) and (20), respectively.

$$
\begin{aligned}
Q^{*} & =y(e)+F^{-1}\left(\frac{p-c+g-m e}{p-v+g}\right) \\
& =\beta-\gamma e+A+N(p-c+g-m e), \\
e^{*} & =\frac{m H+G-\alpha e_{o}}{2 m \gamma+N m^{2}-\alpha} .
\end{aligned}
$$

Here, $N=(B-A) /(p-v+g), H=\beta+A+N(p-c+g)$, and $G=\gamma(p-c+g)$.

\section{Impacts of Carbon Trading Price on Carbon Emissions}

In order to discuss the impacts of carbon trading price on carbon emissions, we assume that the per-unit product carbon emission $e$ is the function of carbon trading price $m$, namely, $k(m)$. From (20), we can get

$$
\begin{aligned}
& \frac{d k(m)}{d m}=-\frac{H N m^{2}+2 m N\left(G-\alpha e_{o}\right)+\alpha H+2 \gamma G-2 \gamma \alpha e_{o}}{\left(2 m \gamma+N m^{2}-\alpha\right)^{2}}, \\
& \frac{d k^{2}(m)}{d m^{2}} \\
& =\frac{4(\gamma+m N)\left[H N m^{2}+2 m N\left(G-\alpha e_{o}\right)+\alpha H+2 \gamma G-2 \gamma \alpha e\right]}{\left(2 m \gamma+N m^{2}-\alpha\right)^{4}} \\
& -2 N \frac{H m+G-\alpha e_{o}}{\left(2 m \gamma+N m^{2}-\alpha\right)^{2}} .
\end{aligned}
$$

When $\left(N m^{2}+\alpha\right) / 2(\gamma+m N)>\sqrt{\gamma^{2}+N \alpha}-\gamma$, we can get Theorem 1.

Theorem 1. When $0<e_{0}<\left(N G-H \gamma+H \sqrt{\gamma^{2}+N \alpha}\right) / N \alpha$,

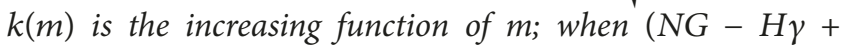
$\left.H \sqrt{\gamma^{2}+N \alpha}\right) / N \alpha<e_{0}<\left(H N m^{2}+2 m N G+\alpha H+2 \gamma G\right) /$ $2 \alpha(\gamma+m N), k(m)$ is also the increasing function of $m$; when $e_{0}>\left(H N m^{2}+2 m N G+\alpha H+2 \gamma G\right) / 2 \alpha(\gamma+m N), k(m)$ is the decreasing function of $m$.

Proof. To solve $d k(m) / d m=0$, one of the necessary and sufficient conditions is $\alpha \neq 2 m \gamma+N m^{2}$. When $\alpha \neq 2 m \gamma+$ $N m^{2}$, if $d k(m) / d m=0$ has real number solutions, then its discrimination should satisfy $\triangle=b^{2}-4 a c \geq 0$, so we can get

$$
\begin{aligned}
& N \alpha^{2} e_{o}^{2}+2 \alpha e_{o}(H \gamma-N G)+N G^{2}-\alpha H^{2}-2 H \gamma G \\
& \quad \geq 0 .
\end{aligned}
$$

We can further solve (23) and get two roots of $e_{0}: e_{0}^{1}=$ $\left(N G-H \gamma-H \sqrt{\gamma^{2}+N \alpha}\right) / N \alpha$ and $e_{0}^{2}=(N G-H \gamma+$ $\left.H \sqrt{\gamma^{2}+N \alpha}\right) / N \alpha$. Due to $N G-H \gamma-H \sqrt{\gamma^{2}+N \alpha}=-\gamma(A+$ $\beta)-H \sqrt{\gamma^{2}+N \alpha}<0$, the root $e_{0}^{1}=(N G-H \gamma-$ 
$\left.H \sqrt{\gamma^{2}+N \alpha}\right) / N \alpha$ is meaningless. So, in (23), there exits only one nonnegative root $e_{0}^{2}=\left(N G-H \gamma+H \sqrt{\gamma^{2}+N \alpha}\right) / N \alpha$.

From (21), it also can be seen that $d k(m) / d m$ is the increasing function of $e_{0}$, and when $e_{0}=\left(\mathrm{HNm}^{2}+2 m N G+\right.$ $\alpha H+2 \gamma G) / 2 \alpha(\gamma+m N), d k(m) / d m=0$. So when $0<e_{0}<$ $\left(N G-H \gamma+H \sqrt{\gamma^{2}+N \alpha}\right) / N \alpha$, we can get $d k(m) / d m>0$; namely, $k(m)$ is the increasing function of $m$; when (NG $\left.H \gamma+H \sqrt{\gamma^{2}+N \alpha}\right) / N \alpha<e_{0}<\left(H N m^{2}+2 m N G+\alpha H+\right.$ $2 \gamma G) / 2 \alpha(\gamma+m N)$, we can get $d k(m) / d m>0$; namely, $k(m)$ is also the increasing function of $m$; when $e_{0}>\left(\mathrm{HNm}^{2}+\right.$ $2 m N G+\alpha H+2 \gamma G) / 2 \alpha(\gamma+m N)$, we can get $d k(m) / d m<0$; namely, $k(m)$ is the decreasing function of $m$.

According to the initial carbon emissions per-unit product $e_{o}$, we can divide the manufacturer into three types: clean manufacturer $\left(e_{0}<\left(N G-H \gamma+H \sqrt{\gamma^{2}+N \alpha}\right) / N \alpha\right.$, such as the solar power manufacturer), middle manufacturer ((NG$\left.H \gamma+H \sqrt{\gamma^{2}+N \alpha}\right) / N \alpha<e_{0}<\left(H N m^{2}+2 m N G+\alpha H+\right.$ $2 \gamma G) / 2 \alpha(\gamma+m N)$, such as general manufacturing enterprise), and nongreen manufacturer $\left(e_{0}>\left(\mathrm{HNm}^{2}+2 m N G+\alpha H+\right.\right.$ $2 \gamma G) / 2 \alpha(\gamma+m N)$, such as steel manufacturer).

From Theorem 1, we can come to a conclusion that the clean manufacturer's optimal per-unit carbon emissions will increase as the carbon trading price increases, which is consistent with the reality. For the clean manufacturer, its per-unit carbon emissions are generally small, and the total carbon emissions often do not exceed the initial carbon emissions limit $M$ assigned by the government. Due to the marginal utility of carbon emissions reduction, it is more and more difficult for the clean manufacturer to upgrade its production technology to reduce carbon emissions. With the carbon trading price increasing, the clean manufacturer will get more profit through carbon trading. So the clean manufacturer is more likely to reduce carbon emissions reduction investment and get more profit through carbon trading. Similarly, we also come to a conclusion that the nongreen manufacturer's optimal per-unit carbon emissions will decrease as the carbon trading price increases. For the nongreen manufacturer, the per-unit carbon emissions are generally large and the total carbon emissions often exceed the initial carbon emissions limit $M$ assigned by the government. When the initial carbon emissions limit cannot meet the nongreen manufacturer's demand of carbon emissions, the rise of carbon trading price will greatly influence its profit, which means that to upgrade its production technology to control carbon emissions is the best choice for the nongreen manufacturer. So, for the nongreen manufacturer, the production technology will be upgraded to control carbon emissions as the carbon trading price increases.

When $\left(N m^{2}+\alpha\right) / 2(\gamma+m N)>\sqrt{\gamma^{2}+N \alpha}-\gamma$, it means that the carbon trading price has a greater impact on the supply chain members' profit than the cost coefficient of carbon emissions reduction. For the middle manufacturer, its per-unit carbon emissions are usually between that of the clean manufacturer and the nongreen manufacturer, which is close to the initial carbon emissions limit $M$ assigned by the government. As a result, when the carbon trading price has a greater impact on its profit, the middle manufacturer is more likely to get profit through carbon trading; when the impact of carbon trading price on its profit is getting smaller, the middle manufacturer is more likely to upgrade production technology to control carbon emissions, same as the nongreen manufacturer. It should be noted that the middle manufacturer is more susceptible to carbon trading price than the clean manufacturer.

When $\left(N m^{2}+\alpha\right) / 2(\gamma+m N) \leq \sqrt{\gamma^{2}+N \alpha}-\gamma$, we can get Theorem 2.

Theorem 2. When $0<e_{0}<\left(H N m^{2}+2 m N G+\alpha H+\right.$ $2 \gamma G) / 2 \alpha(\gamma+m N), k(m)$ is the increasing function of $m$; when $\left(H N m^{2}+2 m N G+\alpha H+2 \gamma G\right) / 2 \alpha(\gamma+m N)<e_{0}<(N G-$ $\left.H \gamma+H \sqrt{\gamma^{2}+N \alpha}\right) / N \alpha, k(m)$ is the decreasing function of $m$; when $e_{0}>\left(N G-H \gamma+H \sqrt{\gamma^{2}+N \alpha}\right) / N \alpha, k(m)$ also is the decreasing function of $m$.

Proof. The proof is similar to the proof Theorem 1. To solve $d k(m) / d m=0$, one of the necessary and sufficient conditions is $\alpha \neq 2 m \gamma+N m^{2}$. When $\alpha \neq 2 m \gamma+N m^{2}$, we can get only one real number root $e_{0}=\left(N G-H \gamma+H \sqrt{\gamma^{2}+N \alpha}\right) / N \alpha$.

From (21), we can see that $d k(m) / d m$ is the decreasing function of $e_{0}$, and when $e_{0}=\left(H N m^{2}+2 m N G+\alpha H+\right.$ $2 \gamma G) / 2 \alpha(\gamma+m N), d k(m) / d m=0$. So when $0<e_{0}<$ $\left(H N m^{2}+2 m N G+\alpha H+2 \gamma G\right) / 2 \alpha(\gamma+m N), d k(m) / d m>0$; namely, $k(m)$ is the increasing function of $m$; when $\left(\mathrm{HNm}^{2}+\right.$ $2 m N G+\alpha H+2 \gamma G) / 2 \alpha(\gamma+m N)<e_{0}<(N G-H \gamma+$ $\left.H \sqrt{\gamma^{2}+N \alpha}\right) / N \alpha, d k(m) / d m<0$; namely, $k(m)$ is the decreasing function of $m$; when $e_{0}>(N G-H \gamma+$ $\left.H \sqrt{\gamma^{2}+N \alpha}\right) / N \alpha, d k(m) / d m<0$; namely, $k(m)$ also is the decreasing function of $m$.

In this condition, we also can divide the manufacturer into three types: clean manufacturer $\left(e_{0}<\left(\mathrm{HNm}^{2}+2 m N G+\right.\right.$ $\alpha H+2 \gamma G) / 2 \alpha(\gamma+m N))$, middle manufacturer $\left(\left(\mathrm{HNm}^{2}+\right.\right.$ $2 m N G+\alpha H+2 \gamma G) / 2 \alpha(\gamma+m N)<e_{0}<(N G-H \gamma+$ $\left.\left.H \sqrt{\gamma^{2}+N \alpha}\right) / N \alpha\right)$, and nongreen manufacturer $\left(e_{0}>(N G-\right.$ $\left.\left.H \gamma+H \sqrt{\gamma^{2}+N \alpha}\right) / N \alpha\right)$.

From Theorem 2, we can come to a conclusion that the decisions of clean manufacturer and nongreen manufacturer do not change when $\left(N m^{2}+\alpha\right) / 2(\gamma+m N) \leq \sqrt{\gamma^{2}+N \alpha}-\gamma$; the reason is same as what is mentioned above. Meanwhile, for the middle manufacturer, the decision behavior has changed. When $\left(N m^{2}+\alpha\right) / 2(\gamma+m N) \leq \sqrt{\gamma^{2}+N \alpha}-\gamma$, it means that the cost coefficient of carbon emissions reduction has a greater impact on the middle manufacturer's profit than the carbon trading price. It is the best choice to upgrade the production technology to control carbon emissions for the middle manufacturer. It also should be noted that the middle manufacturer is more susceptible to the cost coefficient of carbon emissions reduction than the nongreen manufacturer. 
TABLE 1: The parameters of the low carbon supply chain.

\begin{tabular}{llcccccccccc}
\hline Parameters & $v$ & $c_{r}$ & $c_{m}$ & $g_{r}$ & $g_{m}$ & $p$ & $A$ & $B$ & $\beta$ & $\alpha$ & $e_{0}$ \\
\hline Value & 2 & 3 & 3 & 1 & 2 & 35 & 50 & 100 & 120 & 15 & 40 \\
\hline
\end{tabular}

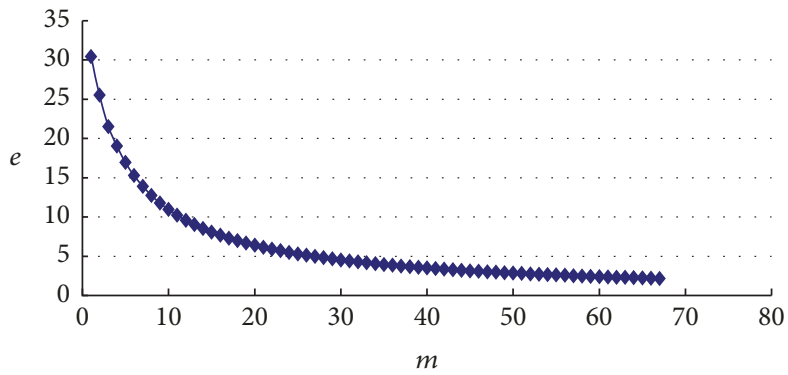

Figure 2: The impacts of carbon trading price on the carbon emissions.

\section{Numerical Experiment}

For the sake of simplicity, we only discuss the impacts of carbon trading price and CEA on the carbon emissions perunit product. The parameters of low-carbon supply chain are shown in Table 1.

According to (20), the optimal per-unit product's carbon emissions of the supply chain can be rewritten as $e^{*}=$ $(214.44 m+32 \gamma-600) /\left(2 m \gamma+1.39 m^{2}-15\right)$.

Firstly, we discuss the impacts of carbon trading price on the carbon emissions per-unit product, here assuming $\gamma=1$. Then we can get $e_{0}=40>(N G-H \gamma+$ $\left.H \sqrt{\gamma^{2}+N \alpha}\right) / N \alpha=39.2$, which means the manufacturer is the nongreen manufacturer, whether $\left(\mathrm{Nm}^{2}+\alpha\right) / 2(\gamma+m N)>$ $\sqrt{\gamma^{2}+N \alpha}-\gamma$ or $\left(N m^{2}+\alpha\right) / 2(\gamma+m N) \leq \sqrt{\gamma^{2}+N \alpha}-\gamma$. Figure 2 shows the impacts of carbon trading price on the carbon emissions per-unit product.

From Figure 2, we can see that the carbon emissions perunit product falls as the carbon trading price increases for the nongreen manufacturer. This is because the nongreen manufacturer often exceeds the initial carbon emissions limit assigned by the government. It is the best choice for the nongreen manufacturer to upgrade the production technology with the rise of carbon trading price. It also can be seen that the downward trend of the carbon emissions perunit product is gradually slow, because the space to reduce carbon emissions is getting smaller and smaller when the carbon emissions level falls to a certain extent.

Then we discuss the impacts of CEA on the carbon emissions per-unit product, here assuming $m=5$. Figure 3 shows the impacts of CEA on the carbon emissions per-unit product.

From Figure 3, we can see that as the sensitive coefficient of the market demand to the product's carbon emissions $\gamma$ increases, the product's carbon emissions decrease. This is because the consumers have environmental awareness; they prefer low-carbon products. If the supply chain members want to win in the competitive market competition, they have

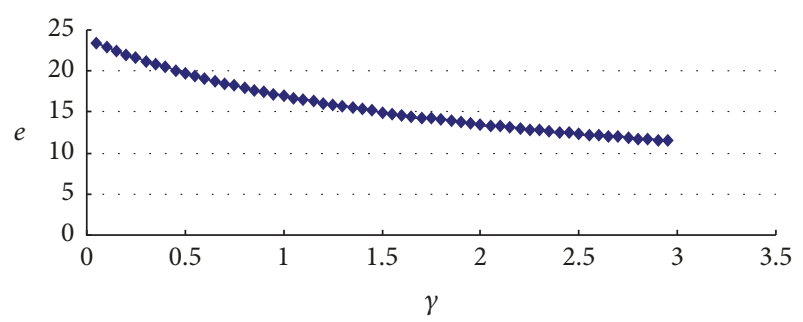

FIgURE 3: The impacts of CEA on the carbon emissions.

to reduce the product's carbon emissions to meet CEA. It also can be seen that the downward trend of the carbon emissions per-unit product is gradually slow as CEA increases. The reason is the same as the impacts of carbon trading price on the carbon emissions.

\section{Conclusions}

Carbon emissions reduction in supply chain is an important way to control carbon emissions to deal with the climate warming. In this paper, we study the carbon emissions reduction in supply chain under cap-and-trade system, in which the demand is stochastic and dependent on the products' carbon emissions. When the low-carbon supply chain coordination is achieved, we also analyze the impacts of carbon trading price on carbon emissions. The conclusions are as follows.

(1) The traditional revenue-sharing contract cannot coordinate the low-carbon supply chain. Because the manufacturer bears the carbon emissions reduction cost and demand is influenced by the products' carbon emissions, the manufacturer and the retailer make the decisions (per-unit carbon emissions and order quantity) to maximize his own profit, not equal to the optimal decisions of the whole supply chain. Meanwhile the traditional revenue-sharing contract does not take these into account and cannot coordinate the low-carbon supply chain.

(2) The improved revenue-sharing contract based on quantity discount policy can coordinate low-carbon supply chain, which achieves the dual goal of economic development and carbon emissions reduction. From (12), we can get $\prod_{r}^{c}(Q)=\psi\left(\prod_{t}(Q, e)+g \mu\right)-g_{r} \mu$ and $\prod_{m}^{c}(Q, e)=(1-$ $\psi)\left(\prod_{t}(Q, e)+g \mu\right)-g_{m} \mu$, which means the retailer's order quantity and the manufacturer's carbon emissions per-unit product equal the optimal order quantity and the carbon emissions per-unit product of the supply chain. Here $\psi=$ $\left[\phi(p-v)+g_{r}\right] /(p-v+g)$.

(3) The change of relationship between the carbon trading price and the cost coefficient of carbon emissions reduction does not affect the decisions of the clean manufacturer and the nongreen manufacturer. The clean manufacturer's perunit carbon emissions increase as the carbon trading price 
increases, which means the clean manufacturer is more likely to get profit through carbon trading, while the nongreen manufacturer's per-unit carbon emissions decrease as the carbon trading price increases, which means the nongreen manufacturer is more likely to get profit through upgrading the production technology.

(4) The change of relationship between the carbon trading price and the cost coefficient of carbon emissions reduction affects the middle manufacturer's decisions. When the carbon trading price has a greater impact on the supply chain members' profit than the cost coefficient of carbon emissions reduction does, the per-unit carbon emissions increase as the carbon trading price increases; when the cost coefficient of carbon emissions reduction has a greater impact on the supply chain members' profit than the carbon trading price, the per-unit carbon emissions decrease as the carbon trading price increases.

Future work can focus on the multicycle situation and the carbon trading cost, which is more common in reality. Also, the model can include carbon trading schemes, where scenarios that have emissions less than the cap can sell back carbon credit and create cash. Such extensions can be important as the Kyoto Protocol is being more and more acknowledged in the global scene.

\section{Conflicts of Interest}

The authors declare that there are no conflicts of interest regarding the publication of this paper.

\section{Acknowledgments}

This research was supported by the Fundamental Research Funds for the Central Universities (nos. 2017B43314, 2015B24014, and 2017B747X14) and Postgraduate Research \& Practice Innovation Program of Jiangsu Province (KYCX170549).

\section{References}

[1] M. X. Wang, Q. Bao, and L. Tang, "Enterprises' optimal abatement investment behavior with the carbon emission constraint," Journal of Management Sciences in China, vol. 18, no. 6, pp. 4157, 2015.

[2] X. H. Chen, X. Y. Zeng, and F. Q. Wang, "Impacts of carbon trading price on carbon emission in supply chain under the capand-trade system," Systems Engineering-Theory \& Practice, vol. 36, no. 10, pp. 2562-2571, 2016.

[3] S. F. Du, L. Hu, and M. Song, "Production optimization considering environmental performance and preference in the cap-and-trade system," Journal of Cleaner Production, vol. 112, no. 20 , pp. 1600-1607, 2016.

[4] A. Braithwaite and D. Knivett, "Evaluating a supply chains carbon footprint: a methodology and case example of carbonto-serve," Logistics Research Network, vol. 11, no. 1, pp. 18-22, 2008.

[5] S. Cholette and K. Venkat, "The energy and carbon intensity of wine distribution: a study of logistical options for delivering wine to consumers," Journal of Cleaner Production, vol. 17, no. 16, pp. 1401-1413, 2009.
[6] B. Sundarakani, R. de Souza, M. Goh, S. M. Wagner, and S. Manikandan, "Modeling carbon footprints across the supply chain," International Journal of Production Economics, vol. 128, no. 1, pp. 43-50, 2010.

[7] H. L. Lam, P. Varbanov, and J. Klemeš, "Minimising carbon footprint of regional biomass supply chains," Resources, Conservation \& Recycling, vol. 54, no. 5, pp. 303-309, 2010.

[8] Q. Xu and D. D. Fan, "Carbon footprint calculation across the whole supply chain," Journal of Donghua University (Natural Science), vol. 40, no. 5, pp. 639-646, 2014.

[9] S. Benjaafar, Y. Li, and M. Daskin, "Carbon footprint and the management of supply chains: Insights from simple models," IEEE Transactions on Automation Science and Engineering, vol. 10, no. 1, pp. 99-116, 2013.

[10] H. Rosič and W. Jammernegg, "The economic and environmental performance of dual sourcing: A newsvendor approach," International Journal of Production Economics, vol. 143, no. 1, pp. 109-119, 2013.

[11] J. Song and M. Leng, "Analysis of the single-period problem under carbon emissions policies," in Handbook of newsvendor problems, vol. 176 of Internat. Ser. Oper. Res. Management Sci., pp. 297-313, Springer, New York, 2012.

[12] S. Du, F. Ma, Z. Fu, L. Zhu, and J. Zhang, "Game-theoretic analysis for an emission-dependent supply chain in a 'cap-andtrade' system," Annals of Operations Research, vol. 228, pp. 135149,2015

[13] M. Y. Jaber, C. H. Glock, and A. M. A. El Saadany, "Supply chain coordination with emissions reduction incentives," International Journal of Production Research, vol. 51, no. 1, pp. 69-82, 2013.

[14] C. Q. Xu, D. L. Zhao, B. Y. Yuan, and L. F. He, "Differential game model on joint carbon emission reduction and low-carbon promotion in supply chains," Journal of Management Science in China, vol. 19, no. 2, pp. 53-65, 2016.

[15] J. Li, Q. Su, and L. Ma, "The research of carbon trading model on supply chain under carbon emission constraints," Chinese Journal of Management Science, vol. 24, no. 4, pp. 54-62, 2016.

[16] D. Z. Zhao, B. Y. Yuan, and C. Q. Xu, "Research on coordination mechanism of the supply chain with the constraint of product carbon emissions," Forecasting, vol. 33, no. 5, pp. 76-80, 2014.

[17] L. Lu and X. Chen, "Supply chain coordination with buyback contract under different carbon emission policies," Control and Decision, vol. 29, no. 12, pp. 2212-2220, 2014.

[18] Z. Liu, T. D. Anderson, and J. M. Cruz, "Consumer environmental awareness and competition in two-stage supply chains," European Journal of Operational Research, vol. 218, no. 3, pp. 602-613, 2012.

[19] L. Zhang, J. Wang, and J. You, "Consumer environmental awareness and channel coordination with two substitutable products," European Journal of Operational Research, vol. 241, no. 1, pp. 63-73, 2015.

[20] Q. P. Wang and D. Z.Zhao, "Revenue-sharing contract of supply chain based on consumer's preference for low carbon products," Chinese Journal of Management Science, vol. 22, no. 9, pp. 106113, 2014.

[21] X. P. Xie, D. Z. Zhao, and Y. J. Liu, "Revenue sharing consignment contract of low-carbon supply chain with carbonemission sensitive demand," Journal of Systems Management, vol. 24, no. 1, pp. 105-117, 2015.

[22] J. Chen, "Study on supply chain management in a low-carbon era," Journal of Systems \& Management, vol. 21, no. 6, pp. 721728, 2012. 
[23] M. Nematollahi, S.-M. Hosseini-Motlagh, and J. Heydari, "Coordination of social responsibility and order quantity in a two-echelon supply chain: A collaborative decision-making perspective," International Journal of Production Economics, vol. 184, pp. 107-121, 2017.

[24] J. Heydari and Y. Norouzinasab, "A two-level discount model for coordinating a decentralized supply chain considering stochastic price-sensitive demand," Journal of Industrial Engineering International, vol. 11, no. 4, pp. 531-542, 2015.

[25] A. Chakraborty and A. K. Chatterjee, "A surcharge pricing scheme for supply chain coordination under JIT environment," European Journal of Operational Research, vol. 253, no. 1, pp. 1424, 2016.

[26] J. Heydari, "Coordinating replenishment decisions in a twostage supply chain by considering truckload limitation based on delay in payments," International Journal of Systems Science, vol. 46, no. 10, pp. 1897-1908, 2015.

[27] X. Feng, I. Moon, and K. Ryu, "Supply chain coordination under budget constraints," Computers \& Industrial Engineering, vol. 88, pp. 487-500, 2015.

[28] J. Heydari, M. Rastegar, and C. H. Glock, "A two-level delay in payments contract for supply chain coordination: The case of credit-dependent demand," International Journal of Production Economics, vol. 191, pp. 26-36, 2017.

[29] S. Ebrahimi, S. Hosseini-Motlagh, and M. Nematollahi, "Proposing a delay in payment contract for coordinating a two-echelon periodic review supply chain with stochastic promotional effort dependent demand," International Journal of Machine Learning and Cybernetics, pp. 1-14, 2017.

[30] J. Heydari, T.-M. Choi, and S. Radkhah, "Pareto improving supply chain coordination under a money-back guarantee service program," Service Science, vol. 9, no. 2, pp. 91-105, 2017. 


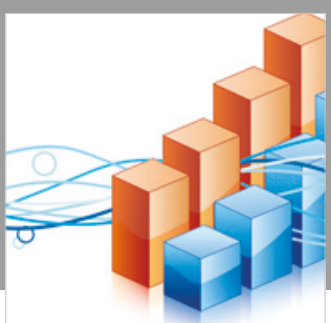

Advances in

Operations Research

\section{-n-m}
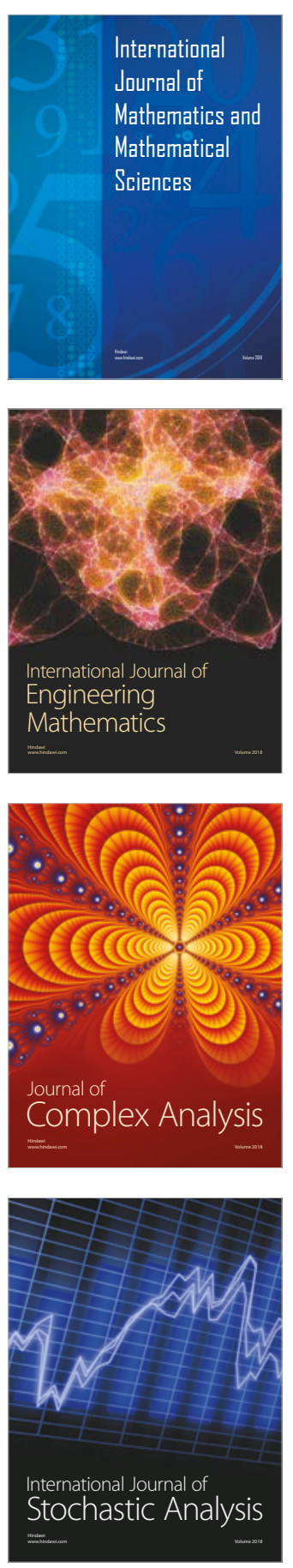
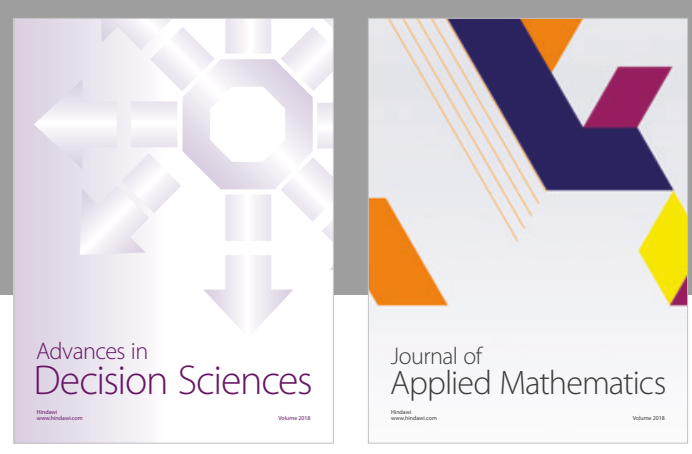

Journal of

Applied Mathematics
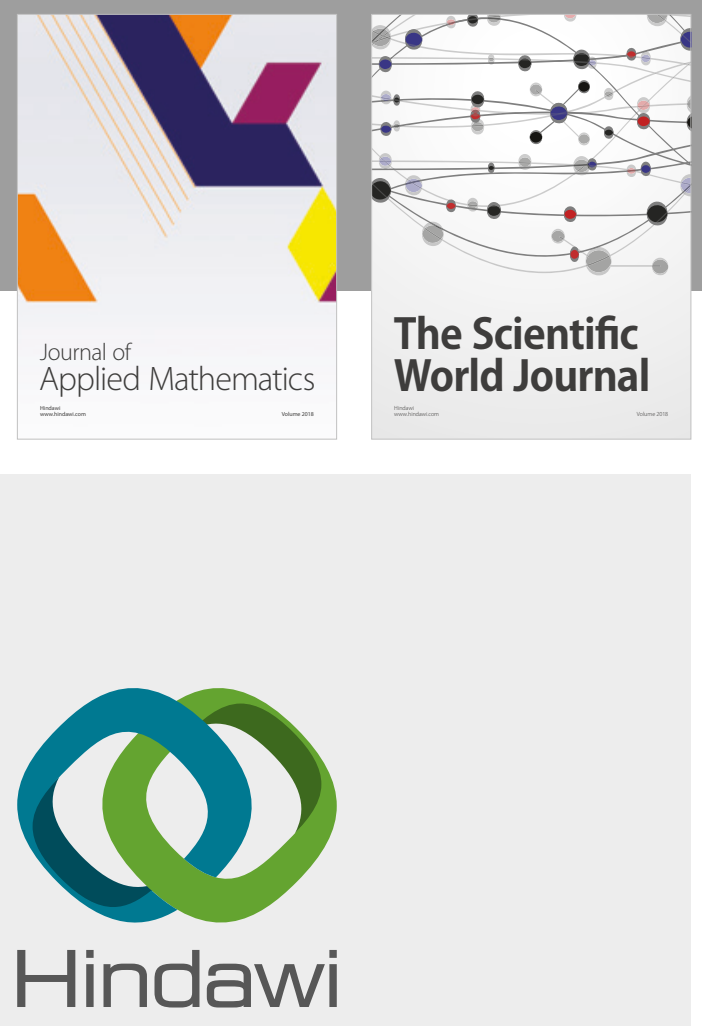

Submit your manuscripts at

www.hindawi.com

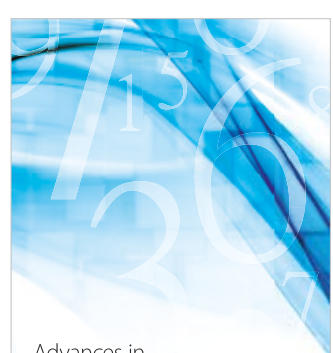

Advances in
Numerical Analysis
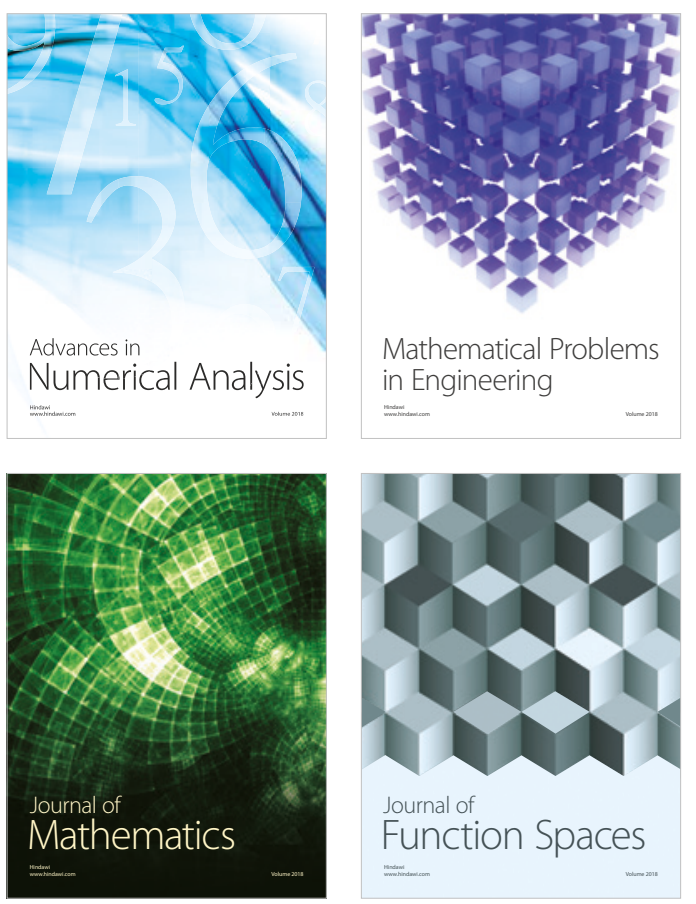

Mathematical Problems in Engineering

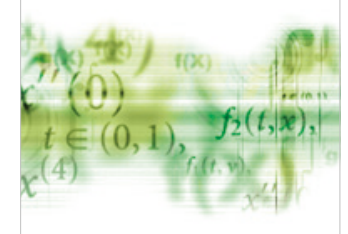

International Journal of

Differential Equations

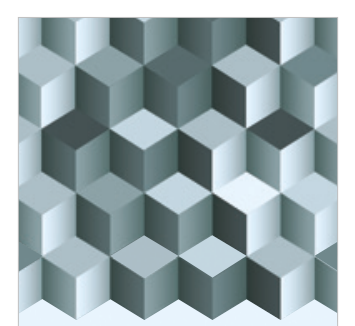

Journal of

Function Spaces

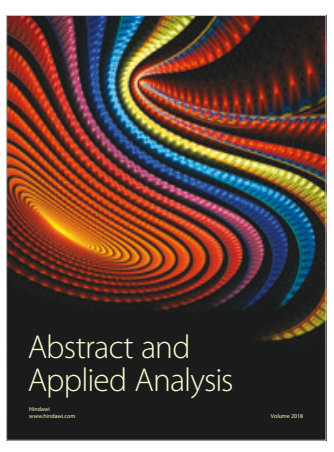

The Scientific

World Journal

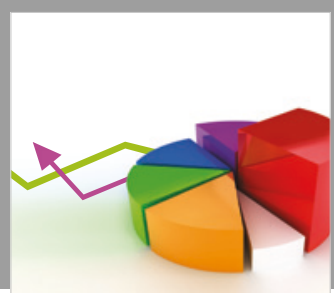

Journal of

Probability and Statistics
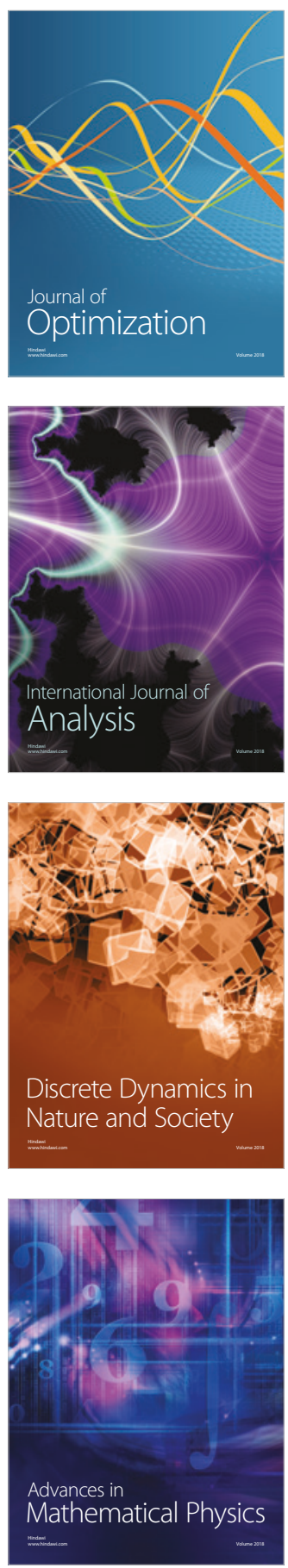